\title{
User Allocation Algorithm with Rate Guarantees for Multi-rate Mobile Networks with Backhaul Constraints
}

\author{
Hiram Galeana, Anna Lainz, and Ramon Ferrús \\ Signal Theory and Communications Department \\ Universitat Politècnica de Catalunya (UPC), Barcelona, Spain. \\ email: \{hiram.galeana, ferrus\}@tsc.upc.edu
}

\begin{abstract}
The optimization of the base station (BS) assignment problem in mobile access networks is a primary task towards enabling efficient utilization of network resources. So far, this problem has been mainly studied in terms of air interface optimization. In this paper, we present a novel BS assignment strategy that integrates backhaul constraints in the user assignment criterion. The motivation of this strategy is the fact that in some scenarios the backhaul can become the network bottleneck. The BS assignment problem or user allocation problem is formulated using a utility-based framework. We take into consideration key aspects such as the revenue associated to each type of service along with the resource consumption in terms of both radio and backhaul resources. Results are given in a multi-service scenario for guaranteed rate services.
\end{abstract}

\section{INTRODUCTION}

The growing popularity of data services in mobile networks requires more advanced strategies to manage the scarcity of radio resources efficiently. Enhanced methods to control users' connectivity to the network play an important role in the radio resource management (RRM) framework as they facilitate an efficient use of radio resources.

This paper addresses the base station (BS) assignment problem that is in charge of determining the serving BS to each user in the system. This problem has been addressed so far mainly from the radio perspective. This is the case of [1][3] that deal with the combined problem of BS assignment and resource allocation, where the primary idea is to determine the assignment of base stations that either minimize the total transmitted uplink power [1], [2] or minimize the sum of the powers of BSs [3]. Furthermore, the problem can be extended to perform power and rate allocation tasks in conjunction with the BS assignment and assuming a maximum transmission power constraint of BSs [4].

The underlying idea of existing BS assignment techniques is that the main limitation in mobile networks is on the air interface and therefore the BS assignment problem has been mainly studied in terms of air interface optimization due that factors like the time-varying system environment and the demand to accommodate mobiles with diverse service requirements make this task challenging. Nevertheless, less attention has been paid to the fact that with the increasing demand of data services and the adoption of enhanced radio technologies backhaul requirements per cell site are drastically growing [5]. Accordingly, and considering that overprovisioning in the backhaul is not an economically feasible option, in some cases the mobile backhaul can become the network bottleneck rather than the air interface.

This work is supported by Spanish National Science Council under grant TEC2006-09109.
Thus, an assignment procedure which is based on radio parameters may assign users to base stations with an overloaded backhaul since its assignment criteria do not consider the backhaul load of BSs. Under this context, in a previous work [6] we proposed an assignment method that allocates users to BSs taking into account the availability of resources in the air interface and the backhaul network. Illustrative results, obtained assuming a single class of mobiles with a fixed transmission rate constraint, indicate that a backhaul-aware strategy lead to a higher number of feasible user assignments.

In this paper we extend the analysis reported in [6] by evaluating the proposed user allocation algorithm in more complex scenarios where data users have varied quality of service (QoS) requirements. We focus on multiservice mobile networks able to guarantee different data rates to users and where bottlenecks can appear in both radio and backhaul segments. The problem consist in assigning users to base stations so that its rate requirement can be fully satisfied, subject to radio and backhaul constraints of each BS. Users that demand different rates are differentiated by means of a profit associated to the corresponding service class. An utility function is defined to quantitatively translate user/operator benefits in terms of the amount of radio resources each user requires and the profit that it will provide to the operator.

The BS assignment problem is formulated as an optimization problem, mapped into a Multiple-Choice Multidimensional Knapsack Problem (MMKP), and then solved using a heuristic search algorithm. The performance of the proposed algorithm is compared to a classical minimum path loss strategy and to a more elaborated strategy that is aimed to optimize the usage of air interface resources but does not account for backhaul resource occupation.

The paper is organized as follows. Next section introduces the system model. Section III presents the formulation of the problem and the utility function defined. The algorithm used to solve the formulated BS assignment problem is described in section IV. Simulation results are provided in section V, and concluding remarks are detailed in section VI.

\section{SYSTEM MODEL}

The problem of assigning data users of different service classes is addressed considering a cellular WCDMA system. Due that high speed services are asymmetric by nature, i.e. bandwidth demand in the downlink is higher than in the uplink, we concentrate in the downlink as it is normally 
viewed as the most restricted link. The considered downlink of a cellular WCDMA system consists of $N$ BSs serving $M$ active users in the entire system. A user assigned to a BS is contained in the matrix $B=\left\{b_{i j}\right\}$, where the indicator variable $b_{i j}=1$ if user $i \in\{1, \ldots, M\}$ is assigned to $\operatorname{BS} j \in\{1, \ldots, N\}$, and zero otherwise. It is assumed that base stations are constrained by the maximum downlink transmission power and the capacity in the backhaul. Therefore, each user requires a percentage of radio and backhaul resources from its serving $\mathrm{BS}$ to fulfill its transmission rate requirement. In order to model the amount of resources been allocated to each user we adopt a cost-based approach as detailed in following subsections.

\section{A. Air Interface Costs}

Let $P_{j}$ be the maximum transmission power of $\mathrm{BS} j$ and $P_{i j}$ the required transmitted power of user $i$ to have a service transmission rate $R_{i}$. The cost associated with the air interface can be expressed as:

$\alpha_{i j}=\frac{P_{i j}\left(R_{i}\right)}{P_{j}}$

Note that this definition entails that the radio cost $\alpha_{i j}$ is coupled with the assignment of all users in the system. This means that the interference reduction associated with the change of the serving BS of one user may result in greater interference to other users [3], causing an increase on their power levels to maintain the transmission rate required. In order to decouple the power computation from the assignment of all users, we can assume that BSs transmit at maximum power. This implies that the power of the interfering BSs for user $i$ is known and therefore the variable factor affecting the perceived interference level is the channel gain of user $i$ with respect to a $\mathrm{BS} j$. This simplification is valid if we assume a fully loaded system and that costs related to the air interface are constant.

\section{B. Backhaul Costs}

In order to model backhaul costs we assume that each BS has a fixed capacity on its backhaul link. In a realistic network deployment such capacity would correspond to the available bandwidth in the path connecting BS $j$ to the upper node (e.g. RNC in 3GPP-based networks), responsible of transporting the aggregated traffic of users being served by $\mathrm{BS} j$. The cost associated with the backhaul network is computed as the ratio of the bit rate required by user $i$ to the available backhaul capacity of its serving $\mathrm{BS}$, denoted as $C_{j}$, as follows:

$\beta_{i j}=\frac{R_{i}}{C_{j}}$

This cost does not depend on the conditions of the rest of users because each user should receive the same transmission rate regardless of its serving $\mathrm{BS}$.

\section{PROBLEM ForMULATION}

The problem of determining the BS assignment solution that satisfies user demands and fulfill network constraints can be expressed in the form of the following optimization problem.

$$
\begin{array}{lll}
\max _{i j}\left(\sum_{i=1}^{M} \sum_{j=1}^{N} u_{i j} b_{i j}\right) & \\
\text { s.t. } & \sum_{i=1}^{M} \alpha_{i j} b_{i j} \leq 1 & j=1, \ldots, N \\
& \sum_{i=1}^{M} \beta_{i j} b_{i j} \leq 1 & j=1, \ldots, N \\
& \sum_{j=1}^{N} b_{i j} \leq 1 & i=1, \ldots, M \\
& b_{i j} \in\{0,1\} &
\end{array}
$$

were $u_{i j}$ in (3) is the utility of user $i$ being assigned to $\mathrm{BS} j$. The set of constraints in (4) and (5) assures that no more resources than available are assigned to each BS. As $\alpha_{i j}$ and $\beta_{i j}$ represent normalized values, the maximum power/backhaul cost supported by each BS is 1 . The third set of constraints in (6) prevents multiple assignments of each user. Finally, in order to avoid splitting or partial assignment of users, constraint (7) is used, which however leads to the combinatorial nature of the problem with exponentially growing complexity in the degrees of freedom.

As argued in [6], the formulated optimization problem is equivalent to a Multiple-Choice Multidimensional Knapsack Problem (MMKP) [7] and a valid option to solve it is through heuristic approaches. The algorithm used to solve the above optimization problem is described in section IV.

\section{A. Utility Function}

Dependent on the location in the service area a user could have a choice of several available BSs. The method to determine which BS should be assigned to each user is based on the notion of utility. The idea is to quantitatively translate user or operator benefits into a utility function that can be seen as a metric taken into account in the assignment process.

Some alternatives to model the utility function are discussed in the following. Assume that the objective is to maximize the total number of assigned users that meet the requested transmission rate. This can be achieved by considering a step function to model the user utility in (3). Hence, $u_{i j}=1$ if the transmission rate of user $i$ is satisfied by assigning it to $\mathrm{BS} j$, or zero otherwise. Under this approach if several feasible solutions exist, any of them is valid as they provide the same maximum value in the objective function, i.e. the total number of users. On the other hand, among the feasible solutions we might want to choose the one that optimize a given network aspect, which in turn should be captured by the utility function. Moreover, in case that no feasible solution exist, the problem is what users should be served, and then aspects like resource costs or the service 
profit can be included into the utility function to serve the most appealing users.

For the above reasons, in this work we consider a utility function that incorporates network related costs and the profit associated with each service. The objective is to mediate between the cost incurred by each user over the air interface and the profit it can provide to the operator, expressed by:

$u_{i j}=\frac{p_{i}}{\alpha_{i j}}$

where $\alpha_{i j}$ is the radio cost of user $i$ being assigned to BS $j$, and $p_{i}$ is the profit of its requested service. In this sense, the utility represents the profit the operator earns per unit of radio resource cost required. The profit can be defined as function of the transmission rate. Consider the case where users $i$ and $i *$ have the same propagation conditions and that $R_{i}>R_{i *}$. In this situation $\alpha_{i j} / \alpha_{i{ }^{*} j}>1$ and in order to give more preference to the user with highest rate it is necessary to satisfy the expression $p_{i} / p_{i^{*}}>\alpha_{i j} / \alpha_{i^{*} j}$. On the other hand, if $R_{i}=R_{i^{*}}$ the prioritization of users will depend on their position in the system, which correspond to different radio resource cost.

\section{User Allocation Algorithm}

Here we briefly describe the heuristic search algorithm employed to solve the BS assignment problem. We assume that the conditions seen by the algorithm do not change, as would be the case of a static system model in which channel gains are assumed to be time-invariant. For a given snapshot of the system the algorithm determines the BS to be assigned to each user so that rate requirements are achieved and radio/ backhaul constraints of all BSs are fulfilled.

The user allocation algorithm is based on the Lagrange Multipliers Theorem proven by Everett [8]. According to this theorem, the constrained optimization problem in (3) can be expressed as an unconstrained optimization problem by associating a multiplier to each radio and backhaul constraint.

Figure 1 shows a schematic representation of the parameters and phases involved in the execution of the algorithm. The algorithm firstly initializes user utilities, radio and backhaul costs, and set Lagrange multipliers to zero. Then, in the Drop phase the algorithm adjusts the assignment of users until it founds a solution that satisfies the constraints of all BSs. During this process the most offending constraint $j^{*}$ is penalized by incrementing the value of the corresponding multiplier. To determine the most appealing BS to each user the algorithm integrates the user utility, radio and backhaul costs and multiplier values into a single measure denominated "weighting utility", expressed as:

$w_{i j}=u_{i j}-\lambda_{j} \alpha_{i j}-\mu_{j} \beta_{i j}$

where $w_{i j}$ is the weighting of utility of user $i$ and BS $j$, while $\lambda_{j}$ and $\mu_{j}$ are the multipliers associated with the radio and transport constraint of $\mathrm{BS} j$, respectively. The role of multipliers is a determining factor in the assignment process due that the higher the occupancy level of radio/backhaul BS resources, the higher the corresponding multiplier value. This avoids that users with high radio/backhaul cost consumption may be assigned to high loaded BSs. The method to adjust the values of Lagrange multipliers is detailed in [6].

Note that the assignment of all users is in general not always possible, e.g. for a large number of users the system could not have enough resources to serve all of them. Thus, in the first phase some users are "dropped" and assigned to a "virtual" BS, corresponding to a utility and resource costs equal to zero. After the execution of Drop phase the assignments satisfies radio and backhaul constraints in all BSs. In the next phase, called $A d d$, the algorithm improves the solution found in Drop phase by means of changing the assignments of those users which can improve the utility in another BS without violating its corresponding constraints. At this point some users could not be accepted to the network due to insufficient radio/backhaul resources. However, as we have assumed that BSs transmit at maximum power, the radio cost obtained for each assignment represents an upper bound. Thus, under real radio cost conditions there may be some space left in the radio constraint of BSs and some users can be allocated. In order to compute the real costs in Relaxation phase we make use of the solution delivered by the Add phase. Users allocated to the virtual BS are assigned to the BS with the highest weighting utility. Then, using the resulting assignment matrix, the power devoted to each user is computed considering the real power values of the interfering BSs, path loss conditions, noise power and user's requirements. Finally, we compute the total aggregated radio and backhaul cost of all BSs and determine the percentage of user that can be serviced without violating both constraints.

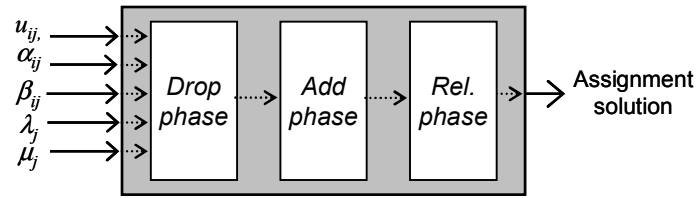

Fig. 1. Schematic representation of the heuristic search algorithm used to determine a BS assignment solution for a given snapshot of the system.

\section{RESUlts}

The performance of the proposed strategy was analyzed considering a cellular deployment with 19 hexagonal cells. A standard wrap around technique is used to avoid border effects. All base stations have a maximum transmission power limited to $43 \mathrm{dBm}$ and a backhaul capacity $C_{j}$ fixed according to the downlink pole capacity $C_{\text {air }}[9]$ by means of a multiplicative factor, denoted as $\phi \in\{1,1.25,1.5, \ldots, 2.5\}$. Hence, $\phi_{j}=1.5$ means that the backhaul capacity of BS $j$ is provisioned with an excess of $50 \%$ of bandwidth over the air interface pole capacity. Main simulation parameters are summarized in Table 1. The provided value of the downlink pole capacity $C_{a i r}$ was computed assuming an average ratio of other-to-own interference $f_{D L}=0.65$ and an average orthogonality factor $\rho=0.5$. In the simulations users are 
uniformly distributed over the overall service area. The BS assignment problem is solved under static conditions (i.e. path loss and shadowing do not change) and repeated 200 times to obtain accurate average values of the percentage of the number of users that can be served while satisfying user's rate requirements and BSs radio and backhaul constraints. The following three BS assignment strategies have been analyzed and compared:

1) Backhaul-aware strategy. This strategy maximizes the utility of all assigned users and guarantees that each user receives the requested transmission rate. If all users cannot be serviced, the strategy discards those users having the worst weighting utility till radio and backhaul constraints are satisfied.

2) Radio-based strategy. Assignments are performed without considering backhaul constraints and therefore the strategy optimize the usage of radio costs by means of user utility maximization. The method to discard users is based on the weighting utility of each user.

3) Minimum Path Loss (MPL) strategy. Users are assigned to the BS with the minimum radio path loss. Here, users are randomly taken out one by one till the BS can serve the remaining users without violating its constraints.

In the first set of simulations we study the performance of the strategies in partially backhaul limited scenarios. That is, a percentage of the BSs is provisioned with a low backhaul capacity, i.e. the ratio of their backhaul capacity to the air interface capacity is $\phi=\{1,1.5\}$, while the rest of BSs do not present backhaul limitations (i.e. $\phi=3$ ). The number of users for each service rate $(128 \mathrm{~K}$ or $384 \mathrm{~K})$ is the same and the profit of both services is set such that $p_{i(128 K)}=p_{i(384 K)}$.

In Fig. 2 we show the gain that radio-based and backhaulaware strategies can achieve over a traditional user allocation method such as MPL. The gain represents the percentage increase of users that these strategies can allocate to the network so that both radio and backhaul constraints are satisfied in the $95 \%$ of the cases. As more BSs have insufficient backhaul capacity, the gain of the backhaul-aware assignment strategy is more pronounced with respect to the benchmark strategies. In such case, the radio-based strategy may assign a user to a BS with exhausted backhaul resources, although there is available backhaul capacity in neighboring BSs. Thereby, an overload situation can take place and consequently rate demands from users could not be fully satisfied. On the other hand, the backhaul-aware strategy take advantage of available backhaul capacity and steer users to neighboring cells with low loaded backhaul/radio constraints.

For instance, when $30 \%$ of the system (about 6 base stations) has limited backhaul capacity the proposed method achieves an improvement of $15 \%$ respect radio-based strategy. When the backhaul of all BSs have sufficient capacity, both radio-based and backhaul-aware approaches behaves similar achieving a gain around $10 \%$ with respect to MPL strategy. In such cases, the major constraint is on the air interface since the
TABLE 1. Simulation PARAMETERS

\begin{tabular}{|l|c|c|}
\hline \multicolumn{1}{|c|}{ Parameter } & \multicolumn{2}{c|}{ Value } \\
\hline \hline Bit rate, $R_{i}$ & $128 \mathrm{Kbps}$ & $384 \mathrm{Kbps}$ \\
\hline$E b / N_{0}$ target & $5.3 \mathrm{~dB}$ & $5.2 \mathrm{~dB}$ \\
\hline Pole capacity, $C_{\text {air }}$ & $1024 \mathrm{Kbps}$ & $1152 \mathrm{Kbps}$ \\
\hline Cell radius & $\mathrm{L}(\mathrm{dB})=128.1+37.6 \log [\mathrm{d}(\mathrm{km})]+\mathrm{S}(\mathrm{dB})$ \\
\hline Propagation model & \multicolumn{2}{|c|}{$10 \mathrm{~dB}$} \\
\hline $\begin{array}{l}\text { Shadowing standard } \\
\text { deviation, } S\end{array}$ & \multicolumn{2}{|c|}{$3.84 \mathrm{Mchips} / \mathrm{s}$} \\
\hline Chip rate, $W$ & \multicolumn{2}{|c|}{$43 \mathrm{dBm}$} \\
\hline BS max. transmitted power & \multicolumn{2}{|c|}{$-101.15 \mathrm{dBm}$} \\
\hline Noise power, $P_{N}$ & \multicolumn{2}{|c|}{} \\
\hline
\end{tabular}

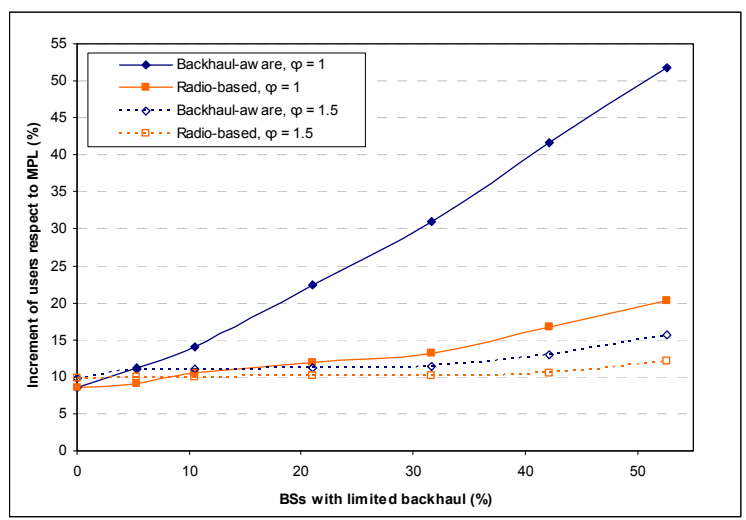

Fig. 2. Percentage increase of Radio-based and Backhaul-aware strategies, respect to the MPL strategy. Two backhaul restrictions are modeled by means of $\phi=1$ and $\phi=1.5$.

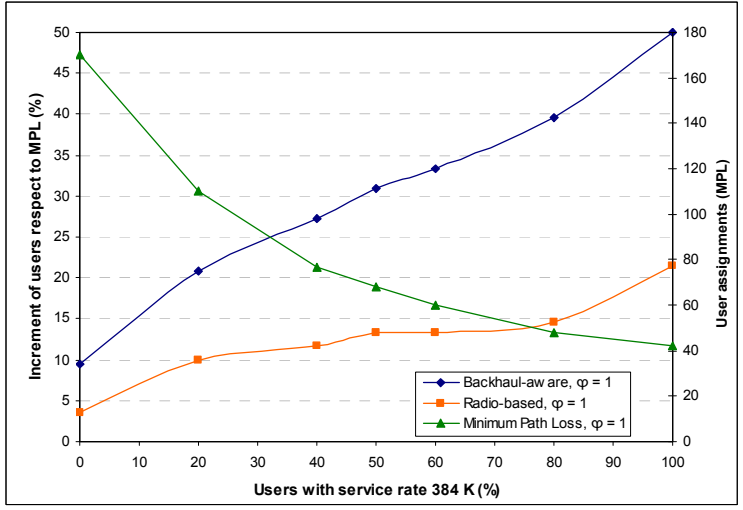

Fig. 3. User assignments' percentage increase respect to MPL versus percentage of total users that request a downlink transmission rate of $384 \mathrm{~K}$. Six BSs in the system have backhaul capacity limitations $(\phi=1)$.

load of backhaul constraints does not normally exceed the maximum capacity of any BS in the system.

In the second set of simulations we study the impact on the performance of the strategies when the distribution of rates required by the users is changed. In this sense, Fig. 3 depicts the total number of users that can be assigned versus the number of users that need to be serviced with a downlink data rate of $384 \mathrm{~K}$. In the left side of the $\mathrm{x}$-axis all users in the service area require to be serviced with a transmission rate of $128 \mathrm{~K}$. From this figure we observe that the gain of the backhaul-aware assignment strategy is more significant for services with high data rate requirements. The ordenate axis on the right of the figure represents the total number of users that MPL strategy can assign without violating radio and 
backhaul constraints in $95 \%$ of the cases.

The impact of the profit value within the utility function over the total number of assigned users per service type is shown in Fig. 4. The distribution of users is set to $50 \%$ for each transmission rate requirement. Clearly, users requiring high transmission rates need more resources from BSs to fulfill their request and they are likely to have low priority in the assignment process. However, we can change this behavior through the profit value assigned to the service requested by a user. In particular, we define the profit ratio $\delta$ of users with $R_{i}=384 \mathrm{~K}$ and $R_{i}=128 \mathrm{~K}$ as $\delta=p_{i(384 \mathrm{~K})} / p_{i(128 \mathrm{~K})}$. If $\delta=1$ the resulting utility of users is exclusively influenced by the radio resource cost. By increasing the profit ratio to 3 we can treat users equally since the radio resource cost incurred by a user with $R_{i}=128 \mathrm{~K}$ is around $1 / 3$ of the air interface cost of a user requesting $384 \mathrm{~K}$. Thus, the utility of both services is almost equal and assignments will depend mostly on the BS constraints each strategy considers.

Simulations were carried out for 72 users in the system, in which MPL strategy can successfully assign $128 \mathrm{~K}$ users in $95 \%$ of the cases. Six BSs in the scenario have backhaul capacity limitations. As one might expect, as the profit value of $384 \mathrm{~K}$ users increase, the acceptance ratio of these users is increased as well. For radio-based strategy the increase of $384 \mathrm{~K}$ users comes at the price of a drastically reduction in the percentage of assigned users with transmission rate of $128 \mathrm{~K}$ because backhaul constraints are not taken into account. On the contrary, the backhaul-aware strategy is able to find backhaul capacity in neighboring BSs so that $128 \mathrm{~K}$ users can still be served. This behavior can also be observed in terms of the total revenue the operator can obtain from an assignment strategy. The total revenue is calculated adding the profit of all assigned users and we have seen it increases linearly with the profit ratio. In Fig. 5 we plot the percentage of the expected revenue $^{1}$ that can be achieved by each assignment strategy. The percentage of the expected revenue slightly decays as the profit of $384 \mathrm{~K}$ users increase. This is due that the reduction of $128 \mathrm{~K}$ users is more significant over the percentage of revenue than the increase of the profit ratio.

\section{CONCLUSION}

This paper has presented a BS assignment algorithm that takes into account radio and backhaul constraints in allocating users from different service classes. We concentrate in scenarios where few base stations have limited backhaul capacity. A user from each service class requires to be served with a fixed data rate transmission in the downlink. We use a utility-based framework to quantitatively translate user (e.g. resource consumption) or operator benefits (i.e. revenue) into a utility function. It has been shown that with the presented algorithm users are likely to be attracted towards the BS with less loaded radio and backhaul constraint and the number of user assignment is increased.

\footnotetext{
${ }^{1}$ The revenue that would be obtained if all users are assigned.
}

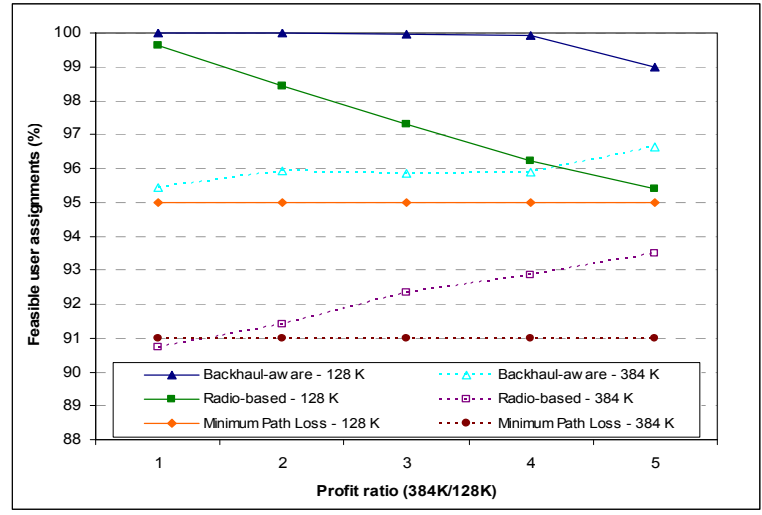

Fig. 4. Feasible user assignment for different profit values of services.

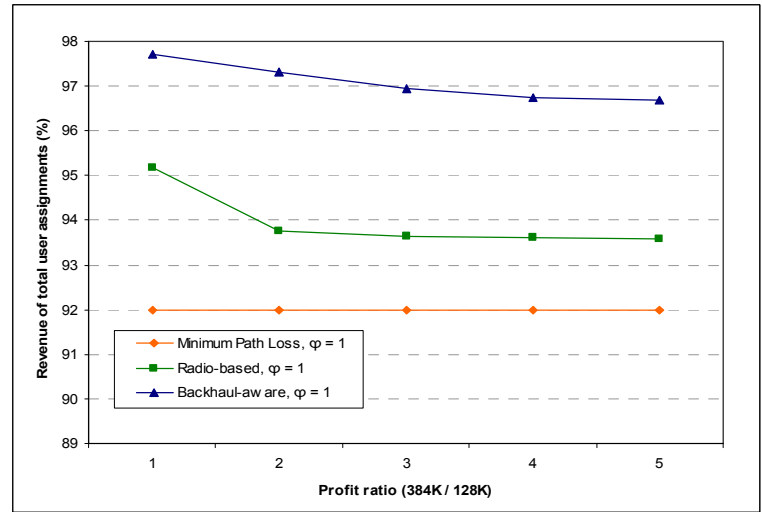

Fig. 5. Percentage of revenue obtained with the assignment of users.

\section{REFERENCES}

[1] R. Yates and C. Y. Huang, "Integrated Power Control and Base Station Assignment," in IEEE Trans. Veh. Technol., vol. 44, Aug. 1995.

[2] R. Yates, "A Framework for Uplink Power Control in Cellular Radio Systems," in IEEE J. Select. Areas in Commun., vol. 13, Sept. 1995.

[3] F. Rashid-Farrokhi, et al, "Downlink Power Control and Base Station Assignment", in IEEE Commun. Letters, vol. 1, no. 4, July 1997.

[4] J. W. Lee, R. R. Mazumdar, N. B. Shroff, "Joint Power Allocation and Base-Station Assignment for the Downlink in CDMA Networks", in IEEE/ACM Trans. On Networking, vol. 14, no. 1, Feb. 2006.

[5] S. Milanovic, "Case Study for Unified Backhaul Performance Optimization", in Journal of Computers, vol. 2, no. 10, December 2007.

[6] H. Galeana, F. Novillo, R. Ferrús, "A Cost-based Approach for Base Station Assignment in Mobile Networks with Backhaul Constraints", in Proc. of Global Communications Conference (GLOBECOM), 2008.

[7] M. Moser, D. P. Jokanovic, and N. Shiratori, "An Algorithm for the Multidimesional Multiple-choice Knapsack Problem" IEICE Transactions Fundamentals Electronics, Communications and Computer Sciences, vol. E80-A, no. 3, March 1997.

[8] H. Everett, "Generalized Lagrange Multiplier Method for Solving Problems of Optimum Allocation of Resources", Operations Research, vol. 2, 1963.

[9] K. Sipila, K. C. Honkasalo, J. L. Steffens, A. Wacker, "Estimation of Capacity and Required Transmission Power of WCDMA Downlink Based on a Downlink Pole Equation", in Proc. of Vehicular Technology Conference (VTC 2000), May 2000. 